\title{
BMJ Open Comparison of health literacy profile of patients with end-stage kidney disease on dialysis versus non-dialysis chronic kidney disease and the influencing factors: a cross-sectional study
}

To cite: Murali K, Mullan J, Roodenrys S, et al. Comparison of health literacy profile of patients with end-stage kidney disease on dialysis versus nondialysis chronic kidney disease and the influencing factors: a cross-sectional study. BMJ Open 2020;10:e041404. doi:10.1136/ bmjopen-2020-041404

- Prepublication history and supplemental material for this paper are available online. To view these files, please visit the journal online (http://dx.doi. org/10.1136/bmjopen-2020041404).

Received 10 June 2020 Revised 05 August 2020 Accepted 16 September 2020

D) Check for updates

(c) Author(s) (or their employer(s)) 2020. Re-use permitted under CC BY-NC. No commercial re-use. See rights and permissions. Published by BMJ.

For numbered affiliations see end of article.

Correspondence to Dr Karumathil Murali; karumathil.murali@health.nsw. gov.au

\section{ABSTRACT}

Objectives Lower health literacy $(\mathrm{HL})$ is associated with poor outcomes in patients with kidney disease. Since HL matches the patient's competencies with the complexities of the care package, the level of HL sufficient in earlier stages of chronic kidney disease (CKD) may be inadequate for patients with end-stage kidney disease (ESKD) on dialysis. We aimed to analyse the $\mathrm{HL}$ profile of patients with ESKD and non-dialysis CKD and examine if there were significant associations with covariates which could be targeted to address $\mathrm{HL}$ deficits, thereby improving patient outcomes.

Design and setting Cross-sectional study of patients with CKD and ESKD from a single Australian health district. Methods We assessed the HL profile of 114 patients with CKD and 109 patients with ESKD using a 44-item multidomain Health Literacy Questionnaire (HLQ) and examined its association with demographic factors (age, gender, race), smoking, income, education, comorbidities, carer status, cognitive function and depression. Using multivariable logistic regression models, $\mathrm{HL}$ profiles of patients with CKD and ESKD were evaluated after adjusting for covariates.

Results Patients with ESKD had similar demographics and educational levels compared with patients with CKD. ESKD had significantly higher frequency of vascular disease, cognitive impairment and depression. Patients with ESKD had better HL scores for the social support domain ( $37.1 \%$ vs $19.5 \%$ in higher HLQ4 tertile, $p=0.004)$, whereas all other $\mathrm{HL}$ domains including engagement with healthcare providers were comparable to CKD. Depression was independently associated with nearly all of the HL domains (HLQ1: OR 2.6, $p=0.030$; HLQ2: OR 7.9, $p=<0.001$; HLQ3: OR 7.6, $p<0.001$; HLQ4: OR 3.5, $\mathrm{p}=0.010$; HLQ5: OR 8.9, $\mathrm{p}=0.001$; HLQ6: OR 3.9, $p=0.002$; HLQ7: OR 4.8, $p=0.001$; HLQ8: OR 5.3, $p=0.001$ ) and education with $\mathrm{HL}$ domains relevant to processing healthrelated information (HLQ8: OR 2.6, $p=0.008$; HLQ9: OR 2.5, $\mathrm{p}=0.006$ ).

Conclusions Despite very frequent interactions with health systems, patients with ESKD on dialysis did not have higher $\mathrm{HL}$ in engagement with health providers and most other HL domains, compared with patients with CKD. Strategies promoting patient-provider engagement and managing depression which strongly associates with lower
Strengths and limitations of this study

- Our study provides a direct comparison of health literacy profile of patients with end-stage kidney disease (ESKD) on dialysis and non-dialysis chronic kidney disease (CKD).

- We used a health literacy instrument with different domains that have independent construct validity and reliability and are directly relevant to the interaction of the patient with health systems and care pathways.

- We used multivariable logistic regression to study the independent effect of ESKD/CKD status and relevant covariates on health literacy.

- The external validity of our results would have improved if our sample size was larger and drawn from multiple geographical locations.

- The reliability of our findings may have been affected by the sensitivity and specificity of the instruments used to measure health literacy and covariates like cognitive function and depression.

HL may address the impact of HL deficits and favourably modify clinical outcomes in renal patients.

\section{INTRODUCTION}

According to the WHO, health literacy (HL) denotes the knowledge, motivation and competence to access, understand, appraise and apply health information, which enables patients to make decisions to maintain or improve their health and quality of life. ${ }^{12}$ Limited HL can impede a patient's ability to interact effectively with health providers, health systems and social networks, thereby negatively impacting on their health outcomes. ${ }^{3}$ In patients with chronic kidney disease (CKD), limited HL has been associated with poor medication adherence, increased rates of hospital admissions, morbidity and mortality ${ }^{45}$ while among 
patients with end-stage kidney disease (ESKD) receiving dialysis, limited HL also contributes to missed dialysis treatments. ${ }^{56}$

Adequate HL enables patients to navigate the complex treatment pathways of kidney diseases including ESKD, which are characterised by a heavy pill burden and the need to adhere to rigorous fluid and dietary restrictions. ${ }^{7}$ The interactive HL framework envisions HL, as a relational concept that matches the skills and abilities of the patient with the demands and complexity of the care package. ${ }^{1}$ Complexity and burden of disease as well as its treatment including diet, fluid, pharmacotherapy and self-care challenges are worse in ESKD compared with CKD. It could be argued, therefore, that the same level of HL sufficient to self-manage earlier stages of CKD may not be adequate to self-manage the more complex treatment challenges experienced by patients with ESKD. It could also be hypothesised that the frequent and regular interactions with the health systems and care providers inherent to dialysis therapy for patients with ESKD have the potential to enhance their HL with respect to patient-provider engagement compared with patients with CKD. However, a meta-analysis evaluating the HL in renal patients found similar prevalence of limited HL among dialysis patients and patients with non-dialysis CKD. ${ }^{8}$ Most of the studies included in this meta-analysis used validated HL tools which assessed the patient's proficiency in health-related vocabulary, rather than capturing the full scope of HL constructs pertinent to chronic disease management. The validated multidomain Health Literacy Questionnaire (HLQ) has been developed to evaluate several HL domains which are directly relevant to the patient's interaction with health systems and delivery of care ${ }^{910}$ A study which used the HLQ reported that dialysis patients have better HL in the domains of social support and engagement with healthcare providers, ${ }^{11}$ when compared with a general patient population. A direct comparison between patients with ESKD and CKD may help delineate the HL patterns and the impact of influencing factors in renal patients.

Based on the gaps in the evidence, we aimed to directly compare the HL profile of patients with ESKD undergoing dialysis with patients with non-dialysis CKD after adjusting for covariates, which included demographic factors, educational level, income, comorbidities, cognitive function and depression. A secondary aim of this study was to examine the independent association of HL with these covariates in patients with CKD and ESKD, in order to identify targets for intervention to address their HL deficits which may improve their health outcomes.

\section{METHODS}

Patients attending the renal outpatient clinics for treatment of CKD and those undergoing in-centre or home dialysis for ESKD, registered in three nephrology units, within an Australian local health district were invited to participate in this cross-sectional study. We aimed to recruit approximately equal numbers of patients with non-dialysis CKD and ESKD undergoing dialysis. Patients were eligible for inclusion if they were 18 years or older, had kidney disease and were able to provide informed consent. Patients were excluded if they had undergone kidney transplantation or were being actively worked up for a living donor transplant; had been diagnosed with dementia or an intellectual impairment; had an anticipated life expectancy of less than 12 months based on the primary nephrologist's assessment; or were on a renal palliative care pathway. In addition, since the test instruments were administered in English, patients who did not speak and read English were excluded.

\section{Patient and public involvement}

Patients or members of the public were not involved in the design, conduct, reporting or dissemination plans of this study as it is not appropriate for the proposed research methods.

\section{Data collection}

Each participant provided informed consent prior to any study procedure. Between January 2015 and June 2016, using a combination of interviewing the participants and reviewing their medical records, we collected demographic data (eg, age, gender, race, educational level and income), information regarding smoking and carer status, as well as clinical data such as cause of renal disease, comorbidities, duration of dialysis in patients with ESKD, serum creatinine and estimated glomerular filtration rate (eGFR) at study enrolment in patients with CKD.

HL was assessed using the 44-item HLQ, which comprehensively assesses the patient's HL skills in different domains. ${ }^{10}$ This instrument assigns four to six test items each to nine independent scales, each scale representing a unique domain of the overall HL construct, each of which has distinct construct validity and reliability. ${ }^{12}$ The scores in each scale are based on the responses to test items in a rating scale with four levels ('strongly disagree', 'disagree', 'agree' or 'strongly agree'-for scales 1-5) or five levels ('cannot do', 'very difficult', 'quite difficult', 'quite easy' or 'very easy'-for scales 6-9). These scales representing the domains include 'feeling understood and supported by healthcare providers' (HLQ1), 'having sufficient information to manage my health' (HLQ2), 'actively managing my health' (HLQ3), 'social support for health' (HLQ4), 'appraisal of health information' (HLQ5), 'ability to actively engage with healthcare providers' (HLQ6), 'navigating the healthcare system' (HLQ7), 'ability to find good health information' (HLQ8) and 'understand health information well enough to know what to do' (HLQ9) ${ }^{10}$ The psychometric properties of the HLQ have been validated in diverse community settings and all HLQ scales were found to be homogeneous with composite reliability ranging from 0.80 to 0.89 , in measuring the relatively narrow and distinct constructs. ${ }^{9}$ 
The modified Mini-Mental State Examination (3MS test) was used to assess cognitive function. ${ }^{13}$ The test assesses a number of domains including memory and fluency, executive function, orientation, visuoconstruction as well as language praxis. The 3MS test has greater sensitivity, specificity, receiver operating characteristics and test-retest reliability than the MMSE. ${ }^{14}$ Patients were categorised as 'cognitively impaired' if their 3MS score was below $85 \%$. The trail-making test (difference between part B and part A) was used to evaluate executive function, ${ }^{15}$ and an interference measure from the Stroop task with the colour word test ${ }^{16}$ was used to assess for cognitive control and flexibility. The Beck Depression Inventory 2 (BDI-2) was used to assess depression, and a cut-off score of $\geq 14$ was used to categorise participants as 'depressed'. ${ }^{17}$ This instrument, which has been translated to 17 languages, has a high degree of reliability with internal consistency around 0.9 and test-retest reliability ranging from 0.73 to 0.96 , with broad applicability for research and clinical practice. ${ }^{18}$

The 3MS test, trail-making test and Stroop task were administered face to face with the study participants by the research nurse. Paper-based copies of the HLQ and BDI-2 questionnaires were handed over to the study participants for completion and return to the research nurse. For patients with ESKD receiving in-centre haemodialysis, test instruments were administered either before or in the first hour of dialysis to avoid any possible fluctuations in cognitive function related to the dialysis process. To avoid patient fatigue, these test instruments were administered in a staggered fashion over consecutive dialysis sessions at the discretion of the research nurse. When the HLQ and BDI-2 questionnaires were returned, the research nurse reviewed their responses and prompted patients to rectify incomplete responses. For patients with non-dialysis CKD, or patients with ESKD receiving homebased dialysis, who attended the outpatient nephrology clinics, all the test instruments and questionnaires were completed in a single session to minimise the need for revisits to the clinics.

\section{Data analysis}

We described baseline characteristics using proportions for discrete variables, and median with IQR for continuous variables. The baseline characteristics were compared between patients with ESKD and CKD using $\chi^{2}$ test for categorical covariates, and non-parametric Wilcoxon rank-sum (Mann-Whitney) test for continuous variables. Cohen's d effect size was used to describe the difference between mean scores of HLQ scales between the ESKD and CKD population. ${ }^{19}$

To evaluate the association of individual HL domains and covariates, we used the categories of higher versus lower HL as the dependent variable in logistic regression models. Since there are no widely agreed threshold HLQ scores to classify patients to the 'higher' or 'lower' HL categories, we divided the population to tertiles for each HL scale and compared the highest versus the lower two tertiles of scores in each HL scale, because tertiles provide a natural order within each domain.

Using a univariate logistic regression model, we examined the effect of independent covariates such as demographic factors including age, gender, indigenous status, education, annual income, carer status, diabetes, depression and cognitive function as well as patient subgroup (CKD vs ESKD) on the dependent variable of HL categories based on tertiles. To minimise bias due to the confounding effect of covariates, we included all covariates that were associated with any of the HLQ scale categories at a significance level of $\mathrm{p}<0.2$ as independent variables, in a multivariable logistic regression model, to ascertain their independent association with each HL domain. We also included the ESKD versus CKD status as an independent variable in the model to identify any significant difference in the HL profile between patients with ESKD and CKD after adjusting for covariates. Missing data were handled by listwise deletion. Statistical significance was set at a two-tailed $p$ value $<0.05$. All the statistical analyses were undertaken using Stata V.16.0.

\section{RESULTS}

\section{Study population}

Of the 321 potentially eligible patients (148 ESKD and 173 CKD) invited to participate in the study, 66 (20.6\%) declined. Five patients were excluded because they did not comply with the inclusion criteria outlined in the Methods section and 27 patients dropped out due to withdrawal of consent, kidney transplantation or death before completing baseline data. The final study cohort comprised 223 patients, 109 with ESKD undergoing dialysis (96 in-centre haemodialysis, 7 peritoneal dialysis, 6 home haemodialysis) and 114 with CKD not receiving dialysis. Figure 1 provides the flow diagram of patient recruitment in the study.

\section{Baseline characteristics}

Table 1 provides the baseline demographic and clinical characteristics of the overall study sample and the two subgroups.

The median age of the population was 69.6 years with the patients with ESKD tending to be younger than the patients with CKD (68.0 years vs 71.0 years). The majority of the patients with ESKD (62\%) and CKD (61\%) were male. Patients with ESKD had significantly lower annual incomes $(p=0.001)$, and more likely to be supported by a personal carer $(\mathrm{p}<0.001)$. Patients with ESKD had a higher prevalence of ischaemic heart disease $(p<0.001)$ and peripheral vascular disease $(p<0.001)$. Educational level, smoking status, aetiology of renal disease, presence of diabetes, hypertension, cerebrovascular disease and lung disease were comparable between the ESKD and CKD population. The median creatinine was $132 \mu \mathrm{mol} / \mathrm{L}$ (IQR 97-171 $\mu \mathrm{mol} / \mathrm{L}$ ) and median eGFR was $41 \mathrm{~mL} / \mathrm{min}$ (IQR $27-57 \mathrm{~mL} / \mathrm{min}$ ) in the patients with CKD at study enrolment. 


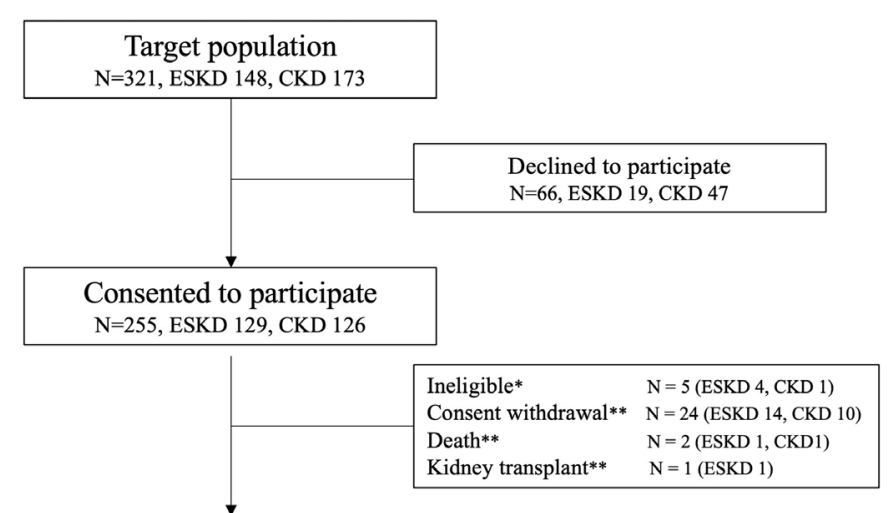

\section{Study population}

$\mathrm{N}=223$, ESKD 109, CKD 114

Figure 1 Flow chart of patient participation in the study. CKD, chronic kidney disease; ESKD, end-stage kidney disease. ${ }^{*}$ neligible patient $(\mathrm{N}=5)$ had dementia (2), intellectual important (1) or anticipated life expectancy $<12$ months due to terminal cancer (1) or severe heart failure (1). ${ }^{*}$ Drop-outs included patients who, after entry into the study, withdrew consent $(\mathrm{N}=24)$, died $(\mathrm{N}=2)$ or had kidney transplantation $(\mathrm{N}=1)$ before full baseline data collection.

Table 2 shows the cognitive and psychological measures in the study sample. Cognitive function assessed by $3 \mathrm{MS}$ test scores $(\mathrm{p}=0.001)$ or as the categorical variable of 'cognitive impairment' $(\mathrm{p}=0.033)$ was significantly worse in patients with ESKD compared with patients with CKD. Cerebral executive function assessed by the difference of part A and part B of the trail-making test was significantly $(p<0.001)$ impaired in patients with ESKD, while the Stroop interference score was not significantly $(p=0.055)$ different between the two groups of patients. Patients with ESKD had a significantly higher frequency of depression compared with patients with CKD $(p<0.001)$.

The HLQ scores for the patients with ESKD and CKD, provided in tables 3 and 4 , highlight that there was no significant difference between the groups, with respect to the mean scores in any of the nine HLQ domains. The Cohen's $d$ showed a small effect size at $0.26(<0.5,>0.2)$ for patients with ESKD in the HLQ2 domain ('have sufficient information to manage health'). Comparisons based on the higher versus lower two tertiles of HLQ scores showed a significantly higher proportion of patients with ESKD in the higher tertile for the 'social support domain' (HLQ4) compared with the patients with CKD $(37.1 \%$ vs $19.5 \%)$. The remaining eight HL domains showed no significant differences.

\section{Association of covariates with HL domains}

The univariate association of the covariates and the categories of higher versus lower two tertiles of HL domains are provided as online supplemental table S1. Patients with ESKD had better HL in the 'social support domain' (HLQ4) compared with patients with CKD (OR 0.41). Depression and education less than 12 years were associated with worse HL in seven and four out of nine domains, respectively.
Results of multivariable logistic regression, including all covariates that showed an association with any HL domain at a significance level of $\mathrm{p}<0.2$ and ESKD versus CKD status as independent variables and HL category as the dependent variable, are given in table 5 . We found that patients with ESKD had significantly better 'social support' HL (HLQ4, OR 0.36) after adjusting for covariates. The association between depression and lower HL was significant for most HL domains (HLQ1-HLQ8) and approached significance for 'understand health information well enough to know what to do' (HLQ9) domain (OR 2.26, $\mathrm{p}=0.051$ ). Lower education was associated with lower 'ability to find good health information' (HLQ8, OR 2.60) and 'understand health information well enough to know what to do' (HLQ9, OR 2.50). Older age was associated with less 'ability to actively manage health' (HLQ3, OR 1.43) and 'ability to find good health information' (HLQ8, OR 1.44). Not having a dedicated carer was significantly associated with lower HL pertaining to 'ability to actively manage health' (HLQ3, OR 2.43) and 'appraisal of health information' (HLQ 5, OR 2.86).

\section{DISCUSSION}

Our study shows that patients with ESKD on dialysis have better HL in domains relevant to 'social support for health' when compared with patients with CKD but not for other domains including 'actively managing health', or 'ability to engage with healthcare providers' despite regular and frequent interactions with health providers and health systems. These findings are counterintuitive and partly contradictory with existing literature. ${ }^{11}$ Our study also highlights the strong association between depression and most HL domains as well as the association between education and HL domains relevant to processing health-related information.

According to the literature, the reported prevalence of limited HL varies from $8.4 \%$ to $49.6 \%$ in patients with ESKD and $16.3 \%$ to $63.3 \%$ in patients with non-dialysis CKD. ${ }^{8}$ The wide variation in the reported prevalence of HL may be due to the variations in the instruments used to measure HL, such as the Single-Item Literacy Screener (SILS), Brief Health Literacy Screener, Short-Test of Functional Health Literacy in Adults, Rapid Estimate of Adult Literacy in Medicine (REALM) and Newest Vital Sign. ${ }^{8}$ Although these commonly used instruments are validated and objective, they measure a very limited set of HL constructs. ${ }^{11}$ We used the more comprehensive and validated $H L Q{ }^{9}$ which assesses the patient's competency in several HL domains that are directly relevant to the interaction between the patient and chronic disease pathways. ${ }^{10}$ Though HLQ is a self-reported instrument and therefore subjective, it has been shown to have measurement veracity at the patient and clinician levels ${ }^{12}$ and covers a greater breadth of the multidimensional concept of $\mathrm{HL}$ as defined by $\mathrm{WHO}^{1}$ compared with the objective HL tools. 
Table 1 Baseline characteristics of the study population comparing patients with non-dialysis CKD and ESKD

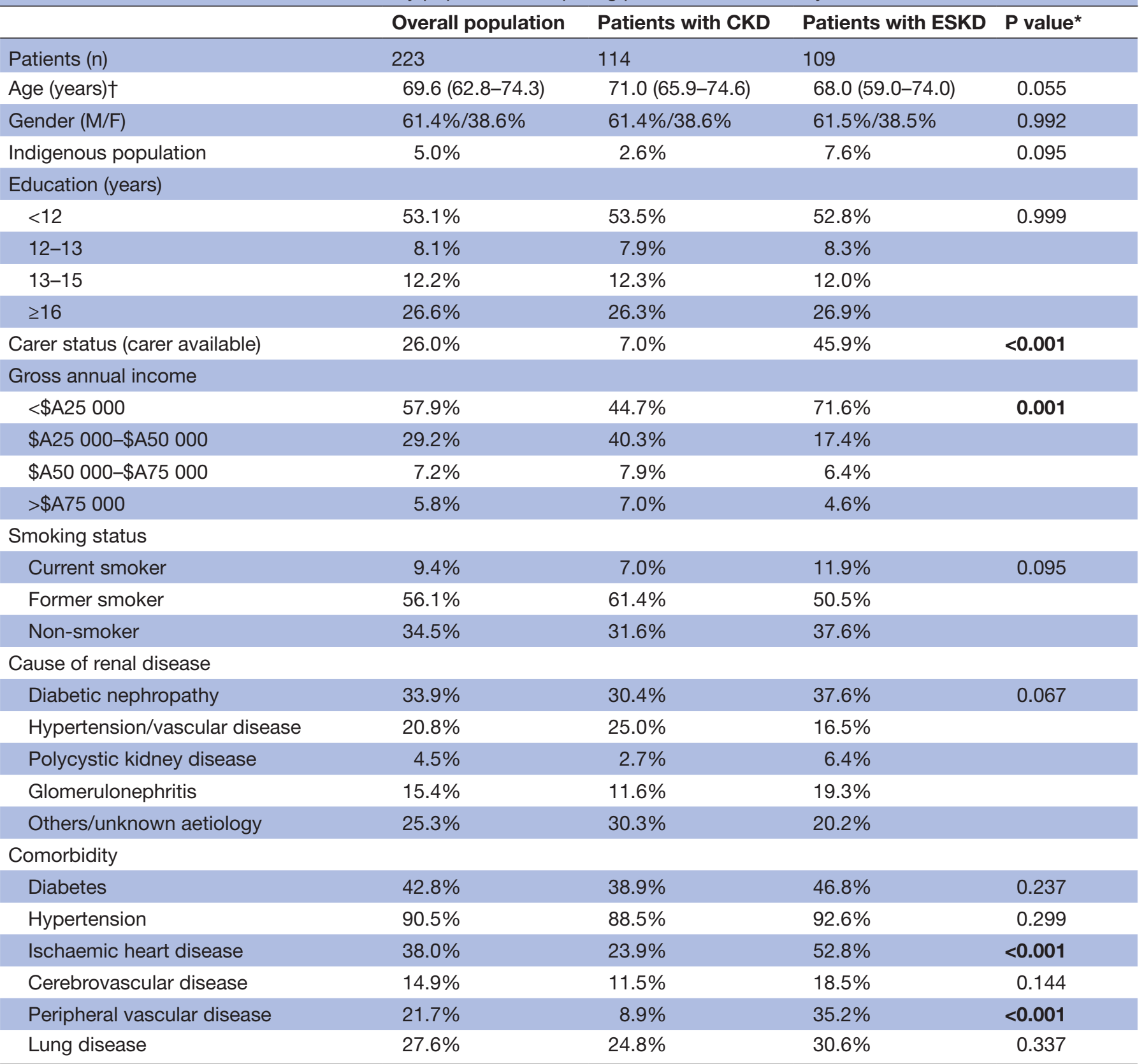

$p$ values with a significance level $<0.05$ are shown in bold format.

${ }^{*} \mathrm{P}$ value for difference estimated by Wilcoxon rank-sum test for continuous variables and by $\chi^{2}$ test for proportions.

†Median and IQR.

CKD, chronic kidney disease; ESKD, end-stage kidney disease; F, female; M, male.

Our observation of the strong independent association of depression with most HL domains highlights the importance of screening for depression in patients with kidney disease. Depression is highly prevalent in this population and associated with higher mortality and diminished quality of life. ${ }^{20}$ Lower HL based on HLQ tool has been shown to be significantly associated with depression in univariate models. ${ }^{11}$ A study using SILS to measure HL reported significant association between limited HL and depression in multivariate models adjusting for demographics, comorbidity and socioeconomic status in the incident dialysis cohort, ${ }^{21}$ while another study adjusting for age, gender, race, educational levels and other clinical variables found no association between depression and limited HL. ${ }^{22}$ In the latter study, HL was assessed using the REALM tool, which focuses on health-related vocabulary and reading comprehension and may not have captured HL domains important in healthcare delivery, such as the ability to appraise health information or engagement with healthcare providers.

We also noted significant associations between educational level and HL domains relevant to 'ability to find 
Table 2 Baseline cognitive and psychological measures of the study population comparing patients with non-dialysis CKD and ESKD

\begin{tabular}{|c|c|c|c|c|}
\hline & $\begin{array}{l}\text { Overall population } \\
(\mathrm{n}=223) \\
\text { Median (IQR)/\% }\end{array}$ & $\begin{array}{l}\text { Patients with } \\
\text { CKD } \\
(n=114) \\
\text { Median (IQR)/\% }\end{array}$ & $\begin{array}{l}\text { Patients with } \\
\text { ESKD } \\
\text { (n=109) } \\
\text { Median (IQR)/\% }\end{array}$ & P value* \\
\hline $\begin{array}{l}\text { Modified Mini-Mental State Examination (3MS) } \\
\text { score } †\end{array}$ & $94(890-97)$ & $95(91-97)$ & $93(86-96)$ & 0.001 \\
\hline $\begin{array}{l}\text { Cognitive impairment (patients with 3MS score } \\
<85 \% \text { ) }\end{array}$ & $17.6 \%$ & $12.3 \%$ & $23.2 \%$ & 0.033 \\
\hline Trail-making test $A$ (TMT-A) (s) & $38(29-52.5)$ & $35(28-45)$ & $43.5(33-68)$ & $<0.001$ \\
\hline Trail-making test B (TMT-B) (s) & $100(73-143)$ & $82(65-117)$ & $120(83-201)$ & $<0.001$ \\
\hline Difference between TMT-A and TMT-B (s) & $62(40-94)$ & $46(33-76)$ & $72(49-129)$ & $<0.001$ \\
\hline Stroop interference score & $-2.5(-7.2$ to 2.8$)$ & $-1.6(-6.1$ to 3.9$)$ & $-3.6(-8.2$ to 1.4$)$ & 0.055 \\
\hline Beck Depression Inventory 2 (BDI-2) score & $9(4-14)$ & $6.5(3-11)$ & $10(5-17)$ & $<0.001$ \\
\hline $\begin{array}{l}\text { Depression (based on BDI-2 score of } 14 \text { or } \\
\text { above) }\end{array}$ & $26.9 \%$ & $14.9 \%$ & $39.5 \%$ & $<0.001$ \\
\hline
\end{tabular}

$p$ values with a significance level $<0.05$ are shown in bold format.

${ }^{*} \mathrm{P}$ value for difference estimated by Wilcoxon rank-sum test for continuous variables and $\chi^{2}$ test for difference in proportions.

†Median and IQR.

CKD, chronic kidney disease; ESKD, end-stage kidney disease.

Table 3 Comparison of mean HLQ scores between patients with CKD and ESKD

\begin{tabular}{|c|c|c|c|c|c|c|}
\hline \multirow[b]{2}{*}{ Domain and description } & \multicolumn{2}{|c|}{ CKD population } & \multicolumn{2}{|c|}{ ESKD Population } & \multirow[b]{2}{*}{ P value* } & \multirow{2}{*}{$\begin{array}{l}\text { Cohen's } \\
\text { d† }\end{array}$} \\
\hline & Mean & $95 \% \mathrm{CI}$ & Mean & $95 \% \mathrm{CI}$ & & \\
\hline $\begin{array}{l}\text { HLQ1 } \\
\text { Feel understood and supported by } \\
\text { healthcare providers }\end{array}$ & 3.27 & 3.18 to 3.35 & 3.32 & 3.23 to 3.42 & 0.251 & 0.12 \\
\hline $\begin{array}{l}\text { HLQ2 } \\
\text { Have sufficient information to manage my } \\
\text { health }\end{array}$ & 2.98 & 2.90 to 3.06 & 3.10 & 3.00 to 3.20 & 0.061 & 0.26 \\
\hline $\begin{array}{l}\text { HLQ3 } \\
\text { Actively managing health }\end{array}$ & 2.96 & 2.88 to 3.03 & 2.91 & 2.81 to 3.01 & 0.622 & -0.10 \\
\hline $\begin{array}{l}\text { HLQ4 } \\
\text { Have social support for health }\end{array}$ & 3.12 & 3.05 to 3.20 & 3.22 & 3.10 to 3.24 & 0.205 & 0.19 \\
\hline $\begin{array}{l}\text { HLQ5 } \\
\text { Appraise health information }\end{array}$ & 2.72 & 2.62 to 2.80 & 2.77 & 2.65 to 2.88 & 0.916 & 0.09 \\
\hline $\begin{array}{l}\text { HLQ6 } \\
\text { Able to engage with healthcare providers }\end{array}$ & 4.18 & 4.10 to 4.26 & 4.13 & 4.02 to 4.25 & 0.964 & -0.09 \\
\hline $\begin{array}{l}\text { HLQ7 } \\
\text { Navigating healthcare system }\end{array}$ & 4.08 & 3.99 to 4.17 & 4.02 & 3.90 to 4.13 & 0.613 & 0.12 \\
\hline $\begin{array}{l}\text { HLQ8 } \\
\text { Ability to find good health information }\end{array}$ & 3.91 & 3.80 to 4.03 & 3.85 & 3.71 to 3.99 & 0.687 & -0.09 \\
\hline $\begin{array}{l}\text { HLQ9 } \\
\text { Understand health information well enough } \\
\text { to know what to do }\end{array}$ & 4.14 & 4.04 to 4.23 & 4.04 & 3.91 to 4.16 & 0.488 & -0.17 \\
\hline
\end{tabular}

$p$ values with a significance level $<0.05$ are shown in bold format

${ }^{*} \mathrm{P}$ value for difference estimated by Wilcoxon rank-sum test for continuous variables and by $\chi^{2}$ test for proportions.

†Cohen's d effect size for difference between scores of patients with ESKD and CKD.

CKD, chronic kidney disease; ESKD, end-stage kidney disease; HLQ, Health Literacy Questionnaire. 
Table 4 Proportion of patients in 'higher' (compared with 'lower' two tertiles) health literacy tertile in CKD and ESKD groups

\begin{tabular}{|c|c|c|c|c|c|}
\hline \multirow[b]{2}{*}{ Domain and description } & \multicolumn{2}{|c|}{ CKD population } & \multicolumn{2}{|c|}{ Dialysis population } & \multirow[b]{2}{*}{ P value* } \\
\hline & $\mathbf{n}$ & $\%$ & $\mathbf{n}$ & $\%$ & \\
\hline $\begin{array}{l}\text { HLQ1 } \\
\text { Feel understood and supported by healthcare } \\
\text { providers }\end{array}$ & 26 & 23.0 & 31 & 29.5 & 0.274 \\
\hline $\begin{array}{l}\text { HLQ2 } \\
\text { Have sufficient information to manage my health }\end{array}$ & 30 & 26.6 & 38 & 36.5 & 0.113 \\
\hline $\begin{array}{l}\text { HLQ4 } \\
\text { Have social support for health }\end{array}$ & 22 & 19.5 & 39 & 37.1 & 0.004 \\
\hline $\begin{array}{l}\text { HLQ5 } \\
\text { Appraise health information }\end{array}$ & 17 & 15.0 & 24 & 22.9 & 0.140 \\
\hline $\begin{array}{l}\text { HLQ8 } \\
\text { Ability to find good health information }\end{array}$ & 33 & 29.2 & 29 & 27.9 & 0.830 \\
\hline $\begin{array}{l}\text { HLQ9 } \\
\text { Understand health information well enough to } \\
\text { know what to do }\end{array}$ & 36 & 31.9 & 34 & 32.7 & 0.896 \\
\hline
\end{tabular}

$p$ values with a significance level $<0.05$ are shown in bold format.

${ }^{*} \mathrm{P}$ value for difference estimated by Wilcoxon rank-sum test for continuous variables and by $\chi^{2}$ test for proportions.

CKD, chronic kidney disease; ESKD, end-stage kidney disease; HLQ, Health Literacy Questionnaire.

good health information' and 'understanding health information enough to know what to do'. Education has been shown to be associated with HL in previous studies ${ }^{22-24}$ which used the REALM tool to measure HL. However, higher HL measured by vocabulary-based tools like REALM was not associated with an increased uptake of self-care behaviours in CKD. ${ }^{25}$ Instruments like the HLQ that directly evaluate specific healthcare delivery-related domains may associate better with self-care behaviour, but this remains unproven and requires further research. The conceptually distinct nature of the HLQ domains ${ }^{11}$ may explain why some domains are sensitive to education while others are not. We also noted that older subjects had lower HL domain scores for 'active management of health' and 'finding good health information'. Multiple comorbidities which are frequent in older patients pose the biggest challenge to active self-management, ${ }^{26}{ }_{27}$ but access to care and affordability also impact the older patients' ability to self-manage their health, especially among culturally and linguistically diverse populations. ${ }^{27}$

Support from a family member or friend acting as a dedicated personal carer can be helpful in navigating the complex care pathways of multimorbidity, accessing and appraising health information, and chronic disease management, especially for the elderly and frail patients. ${ }^{27}$ The Department of Social Services of the Australian Government provides a range of benefits, payments and concessions to eligible carers $^{28}$ to encourage social support for patients suffering from chronic diseases. In our study, patients with ESKD receiving dialysis had significantly higher carer support than patients with CKD. Our finding of significant associations between 'not having a carer' and lower HL for 'actively managing health' and 'appraising health information' may suggest a pathway for how lower social support leads to poor health outcomes. Discrepancy between expected and received social support has been shown to predict mortality in dialysis patients. ${ }^{29}$

Patients with ESKD in our study did not have higher HL in 'engagement with healthcare providers', which is important in the context of frequent and regular interaction of dialysis patients with health providers and systems. Our findings are discrepant to the existing literature which reports better engagement HL in dialysis patients compared with general patient population. ${ }^{11}$ Potential explanations for this discrepancy may be that we compared patients with ESKD with patients with CKD attending the same health service and adjusted for relevant covariates, rather than using a historical control group of diverse patients used in the earlier study. ${ }^{11}$ Patients' capacity to improve their health outcomes can be limited by the lack of knowledge about the disease and its comorbidities, and by psychosocial influences and their inability to effectively interact and communicate with healthcare providers. ${ }^{30}$ Patient engagement and patient activation are integral to the effective delivery 


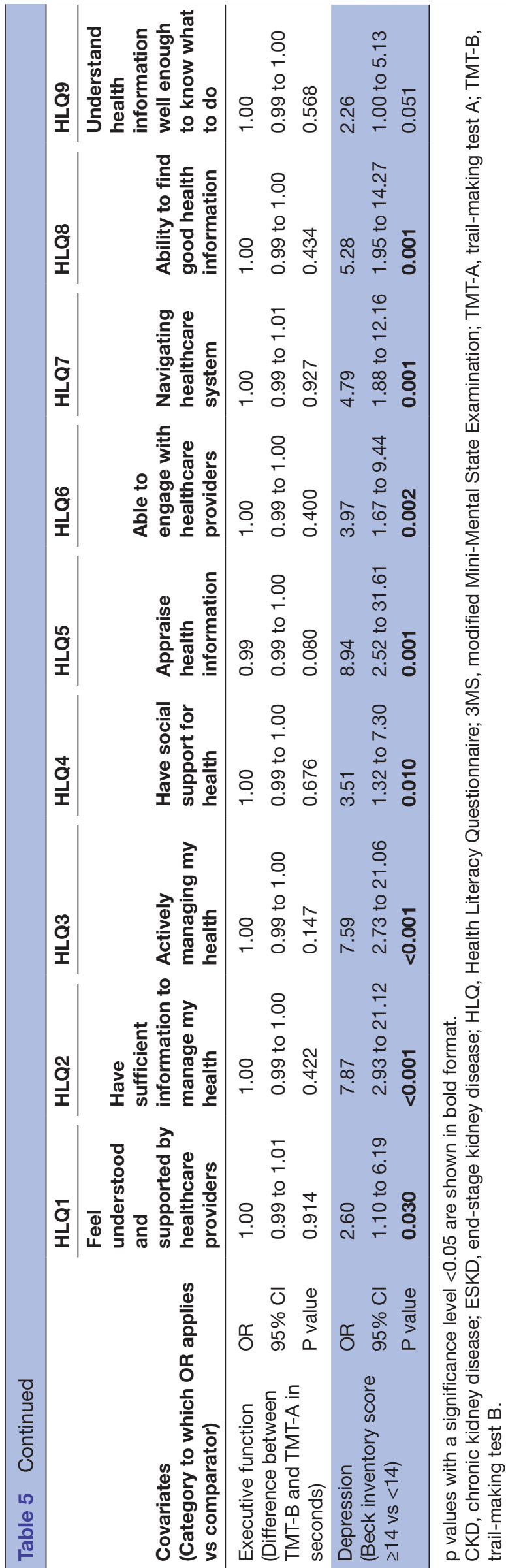

of patient-centred care. Improved treatment adherence has been associated with better patient engagement and activation. ${ }^{31}$ However, it is unclear whether patient-level interventions to enhance these attributes will improve clinical outcomes and requires further research. An integrated approach to address limited HL and support self-management in chronic diseases should engage different stakeholders, including patients, health professionals, healthcare managers, community organisations and policymakers. ${ }^{32} \mathrm{~A}$ systematic review on the effectiveness of patient-level HL interventions reported that most interventions focused on the functional level of HL or numeracy, but the marked heterogeneity in study designs, HL tools and outcome measures compromises the quality of evidence. ${ }^{33}$ On the other hand, health policy initiatives like 'Universal precaution toolkits' help address limited HL by recommending changes to simplify communication and reduce the complexity of healthcare for patients of all HL levels, since the providers may not be able to identify which patients have difficulty in understanding health-related information. ${ }^{34}$ Thus, diverse strategies involving multiple stakeholders are needed to address limited HL. ${ }^{32}$

To our knowledge, this is the first direct comparison of HL profile of patients with ESKD and CKD using a multidomain HL instrument in a robust multivariable model accounting for the relevant confounders. However, our study has several limitations. Selection bias may have occurred in our study because we used a cross-sectional design and almost $30 \%$ of the target population declined to participate or dropped out before the baseline data collection. Our sample size is modest and comprised patients attending renal outpatient clinics or dialysis units in a single health district. A much larger sample from multiple geographical locations would have provided a more confident estimate of the relationships between HL markers and included covariates. However, our use of the comparison group consisting of patients with CKD from the same sociodemographic and geographical cohort provided an opportunity for a more robust comparison between patients with CKD and ESKD after adjusting for confounders. Furthermore, the sensitivity and specificity of instruments used to measure cognitive and psychological measures would have an effect on the reliability of the reported findings. A more robust battery of tests administered by a psychologist may have enabled us to classify cognitive impairment and depression with greater precision, refining the estimate of their association with HL, but such an approach would have limited wider applicability for clinical practice. In our study, the test instruments were administered by research coordinators who were nurses, which is more practical and feasible in a routine clinical setting.

In conclusion, our study highlights the significant association between depression and nearly all the HL domains, while advanced age, educational level and carer support affected specific HL domains. Patients with ESKD on dialysis did not have improved engagement with providers 
compared with patients with CKD after adjusting for other relevant covariates but had improved social support markers, even after adjusting for the presence of a carer. Though the impact of HL interventions in improving the HL metrics is unclear, strategies to address depression, patient-provider engagement and self-management support strategies may help improve health outcomes for patients with ESKD and CKD.

\section{Author affiliations}

'Department of Nephrology, Wollongong Hospital, Wollongong, New South Wales, Australia

${ }^{2}$ Australian Health Services Research Institute, University of Wollongong Faculty of Business, Wollongong, New South Wales, Australia

${ }^{3}$ School of Psychology, University of Wollongong, Wollongong, New South Wales, Australia

${ }^{4}$ Illawarra Health and Medical Research Institute, University of Wollongong, Wollongong, New South Wales, Australia

Acknowledgements The Health Literacy Questionnaire (HLQ) is copyrighted by Drs Richard Osborne, Rachelle Buchbinder, Roy Batterham and Gerald Elsworth (2012). We acknowledge the authors for providing permission to use their instrument. We acknowledge the assistance from School of Psychology, University of Wollongong in providing licensed copies of test instruments (Beck Depression Inventory 2).

Contributors KM contributed to the conception and design of the study, obtaining ethics approval, reviewing patient screening, consenting eligible participants, checking accuracy of collected data, data analysis, interpretation of results, preparation of draft manuscript and revision of manuscript. JM, SR and ML contributed to the conception and design of the study, analysis and interpretation of data and revision of manuscript for important intellectual content. KM, JM, SR and ML were responsible for responding to reviewer comments, approval of the final draft and agree to be accountable for all aspects of the work.

Funding The authors have not declared a specific grant for this research from any funding agency in the public, commercial or not-for-profit sectors.

Competing interests None declared.

Patient consent for publication Not required.

Ethics approval University of Wollongong (UOW) and Illawarra Shoalhaven Local Health District (ISLHD) Health and Medical Human Research Ethics Committee granted ethics approval on 12 November 2014 as per reference number HE14/398-HREC/14/WGONG/90.

Provenance and peer review Not commissioned; externally peer reviewed.

Data availability statement Data are available in a public, open access repository. Extra data can be accessed via the Dryad data repository at http://datadryad.org/ with the doi:10.5061/dryad.2bvq83bnv.

Supplemental material This content has been supplied by the author(s). It has not been vetted by BMJ Publishing Group Limited (BMJ) and may not have been peer-reviewed. Any opinions or recommendations discussed are solely those of the author(s) and are not endorsed by BMJ. BMJ disclaims all liability and responsibility arising from any reliance placed on the content. Where the content includes any translated material, BMJ does not warrant the accuracy and reliability of the translations (including but not limited to local regulations, clinical guidelines, terminology, drug names and drug dosages), and is not responsible for any error and/or omissions arising from translation and adaptation or otherwise.

Open access This is an open access article distributed in accordance with the Creative Commons Attribution Non Commercial (CC BY-NC 4.0) license, which permits others to distribute, remix, adapt, build upon this work non-commercially, and license their derivative works on different terms, provided the original work is properly cited, appropriate credit is given, any changes made indicated, and the use is non-commercial. See: http://creativecommons.org/licenses/by-nc/4.0/.

\section{ORCID iD}

Karumathil Murali http://orcid.org/0000-0003-4827-6981
REFERENCES

1 Kickbusch IPJApfel F, Agis T, eds. Health literacy: the solid facts. 1st edn. Copenhagen: World Health Organisation Regional Office for Europe, 2013.

2 Sørensen K, Pelikan JM, Röthlin F, et al. Health literacy in Europe: comparative results of the European health literacy survey (HLS-EU). Eur J Public Health 2015;25:1053-8.

3 Dageforde LA, Cavanaugh KL. Health literacy: emerging evidence and applications in kidney disease care. Adv Chronic Kidney Dis 2013;20:311-9.

4 Cavanaugh KL, Wingard RL, Hakim RM, et al. Low health literacy associates with increased mortality in ESRD. JASN 2010;21:1979-85.

5 Taylor DM, Fraser S, Dudley C, et al. Health literacy and patient outcomes in chronic kidney disease: a systematic review. Nephrol Dial Transplant 2018;33:1545-58.

6 Green JA, Mor MK, Shields AM, et al. Associations of health literacy with dialysis adherence and health resource utilization in patients receiving maintenance hemodialysis. Am J Kidney Dis 2013;62:73-80.

7 Murali KM, Mullan J, Roodenrys S, et al. Strategies to improve dietary, fluid, dialysis or medication adherence in patients with end stage kidney disease on dialysis: a systematic review and meta-analysis of randomized intervention trials. PLoS One 2019;14:e0211479.

8 Taylor DM, Fraser SDS, Bradley JA, et al. A systematic review of the prevalence and associations of limited health literacy in CKD. CJASN 2017;12:1070-84.

9 Elsworth GR, Beauchamp A, Osborne RH. Measuring health literacy in community agencies: a Bayesian study of the factor structure and measurement invariance of the health literacy questionnaire (HLQ). BMC Health Serv Res 2016;16:508.

10 Osborne RH, Batterham RW, Elsworth GR, et al. The grounded psychometric development and initial validation of the health literacy questionnaire (HLQ). BMC Public Health 2013;13:658.

11 Dodson S, Osicka T, Huang L, et al. Multifaceted assessment of health literacy in people receiving dialysis: associations with psychological stress and quality of life. J Health Commun 2016;21:91-8.

12 Hawkins M, Gill SD, Batterham R, et al. The health literacy questionnaire (HLQ) at the patient-clinician interface: a qualitative study of what patients and clinicians mean by their HLQ scores. BMC Health Serv Res 2017;17:309.

13 Teng EL, Chui HC. The modified Mini-Mental state (3MS) examination. J Clin Psychiatry 1987;48:314-8.

14 Rapp SR, Espeland MA, Hogan P, et al. Baseline experience with modified mini mental state exam: the women's health Initiative memory study (WHIMS). Aging Ment Health 2003;7:217-23.

15 Gordon NG. The TRAIL making test in neuropsychological diagnosis. J Clin Psychol 1972;28:167-9.

16 Graf P, Uttl B, Tuokko H. Color- and picture-word stroop tests: performance changes in old age. J Clin Exp Neuropsychol 1995;17:390-415.

17 Steer RA, Ball R, Ranieri WF, et al. Dimensions of the Beck depression inventory-II in clinically depressed outpatients. J Clin Psychol 1999;55:117-28.

18 Wang Y-P, Gorenstein C. Psychometric properties of the Beck depression Inventory-II: a comprehensive review. Rev. Bras. Psiquiatr 2013;35:416-31.

19 Dodson SGS, Osborne RH. Health literacy toolkit for lowand middle-income countries: a series of information sheets to empower communities and strengthen health systems. New Delhi: World Health Organization, Regional Office for South-East Asia, 2015.

20 Shirazian S, Grant CD, Aina O, et al. Depression in Chronic Kidney Disease and End-Stage Renal Disease: Similarities and Differences in Diagnosis, Epidemiology, and Management. Kidney Int Rep 2017;2:94-107.

21 Taylor DM, Bradley JA, Bradley C, et al. Limited health literacy in advanced kidney disease. Kidney Int 2016;90:685-95.

22 Green JA, Mor MK, Shields AM, et al. Prevalence and demographic and clinical associations of health literacy in patients on maintenance hemodialysis. CJASN 2011;6:1354-60.

23 Jain D, Sheth $H$, Green JA, et al. Health literacy in patients on maintenance peritoneal dialysis: prevalence and outcomes. Perit Dial Int 2015;35:96-8.

24 Lambert K, Mullan J, Mansfield K, et al. A cross-sectional comparison of health literacy deficits among patients with chronic kidney disease. J Health Commun 2015;20:16-23.

25 Schrauben SJ, Cavanaugh KL, Fagerlin A, et al. The Relationship of Disease-Specific Knowledge and Health Literacy With the Uptake of Self-Care Behaviors in CKD. Kidney International Reports 2020;5:48-57. 
26 Bowling CB, Vandenberg AE, Phillips LS, et al. Older Patients' Perspectives on Managing Complexity in CKD Self-Management. CJASN 2017;12:635-43.

27 van Gaans D, Dent E. Issues of accessibility to health services by older Australians: a review. Public Health Rev 2018;39:20.

28 Department of Social services TAG. Supporting carers Canberra, act 2601, 2020. Available: https://www.dss.gov.au/disability-and-carers/ carers

29 Thong MSY, Kaptein AA, Krediet RT, et al. Social support predicts survival in dialysis patients. Nephrol Dial Transplant 2007;22:845-50.

30 Lopez-Vargas PA, Tong A, Phoon RKS, et al. Knowledge deficit of patients with stage 1-4 CKD: a focus group study. Nephrology 2014;19:234-43.
31 Graffigna G, Barello S, Bonanomi A. The role of patient health engagement model (PHE-model) in affecting patient activation and medication adherence: a structural equation model. PLoS One 2017; 12:e0179865.

32 Jordan JE, Briggs AM, Brand CA, et al. Enhancing patient engagement in chronic disease self-management support initiatives in Australia: the need for an integrated approach. Medical Journal of Australia 2008;189:S9-13.

33 Visscher BB, Steunenberg B, Heijmans M, et al. Evidence on the effectiveness of health literacy interventions in the EU: a systematic review. BMC Public Health 2018;18:1414.

34 Brega AG, Freedman MAG, LeBlanc WG, et al. Using the health literacy universal precautions toolkit to improve the quality of patient materials. J Health Commun 2015;20 Suppl 2:69-76. 